\title{
Estimation and Analysis of Harmonics in a Bus System
}

\author{
${ }^{1}$ Kadiyala Chythanya, ${ }^{2}$ Mohammed Tafeel, ${ }^{3} \mathrm{M}$ Dheeraj Sai Krishna, ${ }^{4}$ Dr.S.Ravi Chandran \\ ${ }^{1,2,3}$ UG Student, ${ }^{4}$ Professor, Department of Electrical and Electronics Engineering, Sreenidhi \\ Institute of Science and Technology, Hyderabad, India. \\ 1'chythuk98@gmail.com, ${ }^{2}$ mdtafeel5@gmail.com, ${ }^{3}$ mullapudidheeraj23@gmail.com, \\ 4 csstravichandr@gmail.com
}

\begin{abstract}
Harmonics play an important role in regressing of the power quality. Generally harmonics are generated due to the presence of nonlinear loads and this can counter effect on the system. This paper deals with the estimation and analysis of harmonics in a power line network. In order to estimate the effect, the harmonics are been introduced for the bus system and harmonics analysis is been done. To mitigate the harmonics we use harmonic filters. We are using MiPower software to resolve this problem as this software has no limit on the number of buses in the system and is highly interactive. In this project, five bus systems is designed using MiPower software where harmonics have been injected to the buses and its effects are studied.
\end{abstract}

Keywords - Power quality, MiPower software, Load flow analysis, Harmonic analysis, Total Harmonic Distortion (THD)

\section{INTRODUCTION}

In an electric power system, the harmonics means that current and voltage are been cockeyed and drifted from the sinusoidal wave. These are generated by the nonlinear loads such as rectifiers, discharge lighting, variable speed drivers, transistors etc. A load is said to be non-linear when the supply voltage does not match with the current it draws, where as the voltage harmonics are mostly caused due to current harmonics. Voltage harmonics are in miniature when correlated with current harmonics. The nonlinear loads majorly produce harmonics which are odd multiples of the fundamental frequency. The individual frequency element that involves a composite waveform is invoked as harmonic number (h). Harmonics lessens the level of power quality and reduces the efficiency of the system. In general, most of the systems can tolerate nonlinear loads up to $15 \%$ of the total electricity system capacity without any trouble [3]. Total harmonic distortion (THD) is an

index of a distortion of a signal and is the proportion among the rms value of all harmonics and rms of the fundamental frequency. The values of active power, reactive power and apparent power are to be illustrated thoroughly. The power factor is affected by the harmonics and also results in conductor losses, skin effect, resonances. It also leads to the damaging of the motors, generators, transformer windings as a result of overheating generated by harmonics [2]. Circuit breakers and fuses are also flawed thus resulting in reduction in the performance of the system. In order to mitigate the harmonics, harmonics filters are used.

\section{MIPOWER SOFTWARE}

MiPower software is an Indian based software and was developed by Dr.Nagrath a power systems engineer. It is a power systems analysis and computer aided software. Graphical User Interface (GUI) and database are the two essential components which are needed for the legitimate execution of network and to design any network. We will link the database with the graphical user interface and this generates a path so that we can enroll values to the components. The main advantage of this software is that the number of buses designed can be unlimited and it takes environmental conditions into the picture. It is userfriendly and highly interactive [3]. This software is used for different purpose of simulation like load flow analysis, harmonic analysis, short circuit analysis, voltage instability, fault occur in a line, bus and type of fault, and economic load dispatch [2],[4].

\section{SIMULATION PROCESS}

A Single Line Diagram for an number of bus systems in MiPower software is drawn by configuring the database manager as a simulation file. Later on we can use the components of the bus system present in the MiPower software. To draw a bus, click on the symbol and draw it horizontally or vertically as required and a bus data window will pop up, where in nominal voltage is to be specified. Transmission line is to be drawn between the 
buses, so click on the icon and draw it from one end to another between the buses and in the transmission data pop window enters the values of De-rated MVA, structure reference number, positive sequence resistance, positive sequence reactance, and positive sequence susceptance accordingly. Now click on the generator icon and give it to the bus and generator data is to be given in the pop up window where in De-rated MVA, real power minimum, real power maximum, reactive power minimum, and reactive power maximum, MVA rating, MW rating, KW rating accordingly. Click on the load icon and attach it to the buses as required and a pop up window appears where load data such as real power in MW, reactive power in MVAR are to be given and compute these two to calculate the power factor. Now a RMS current source is attached to the buses and the harmonics are fed by specifying the harmonic numbers. Here we have injected harmonics to the buses 3, 4, 5. Where load flow analysis and harmonic analysis are studied as follows.

Load flow analysis can be achieved by Gauss seidel, Newton raphson, fast decoupled, DC load flow methods. We have selected Newton raphson method for feasible calculations of iteration method then we have to obtain generation is equal to the sum of load and loss, if not the given data or single line diagram is incorrect [7]. In harmonics analysis where average total harmonic distortion with respect to each bus is plotted against percentage distortion

$[1],[8]$.

\section{PROPOSED SYSTEM}

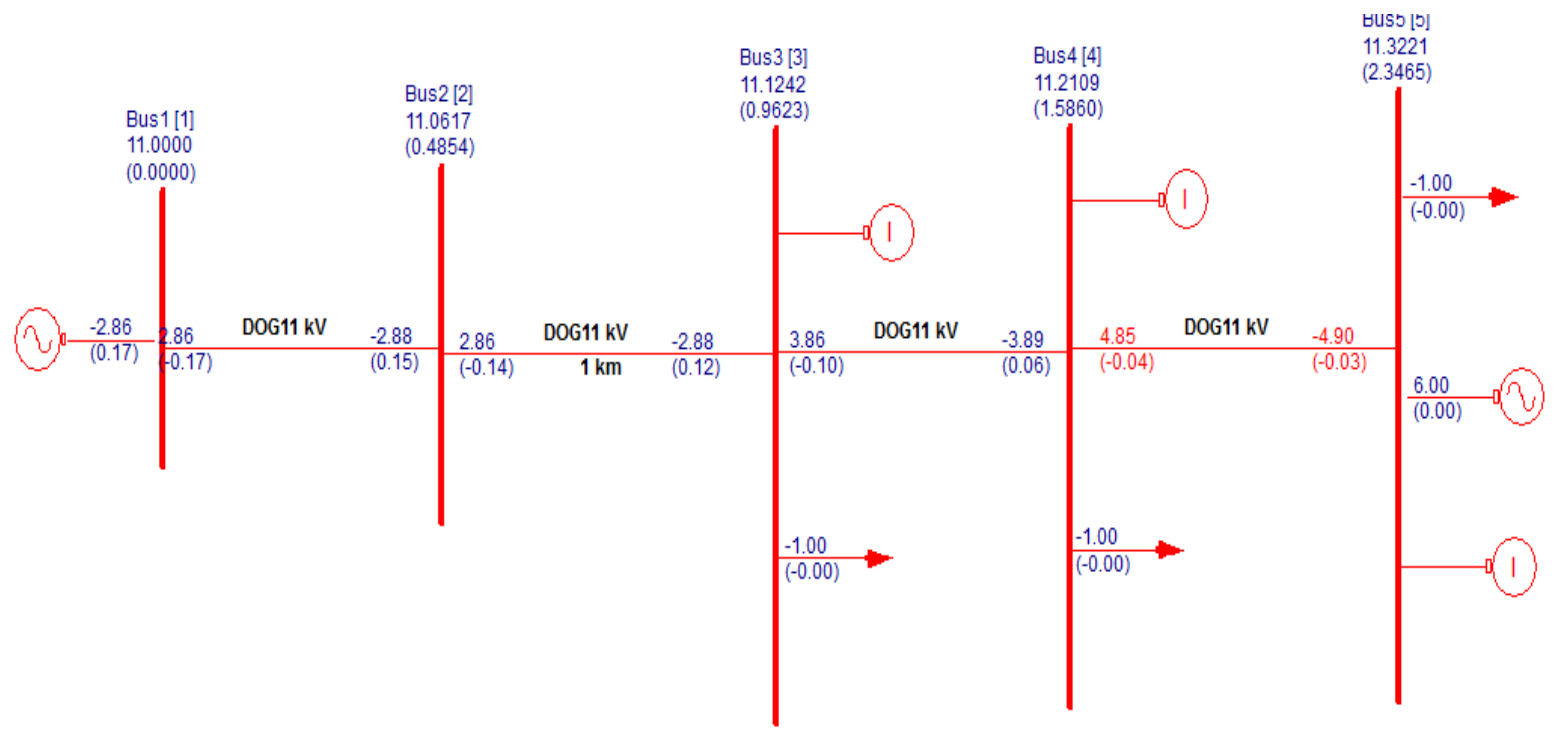

Figure 1 Single Line Diagram for Load flow \& Harmonic Analysis

\section{OUTPUTS}

\section{LOAD FLOW ANALYSIS}

|::::: LOAD FLOW ANALYSIS ::::::|

|----CASE NO: 1 CONTINGENCY: 0 SCHEDULE NO : 0-----|

-----CONTINGENCY NAME: Base Case RATING CONSIDERED:

NOMINAL -----|

$\% \%$ First Power System Network

| INPUT DATA |

|***** SYSTEM SPECIFICATION *****|
Largest Bus Number Used : $\quad 5$

Actual Number of Buses $\quad: 5$

Number of Transmission Lines $\quad: 4$

Number of Generators $\quad: 2$

Number of Loads $\quad: 3$

Number of Zones $\quad: 1$

Base MVA $\quad: 100.0$

Nominal System Frequency $(\mathrm{Hz}) \quad$ : 50.0

Q Checking Limit (Enabled) : 4

Real Power Tolerance (p.u.) : : 0.00100

Reactive Power Tolerance (p.u.) : 0.00100

Maximum Number of Iterations : 15

Bus Voltage below which Load Model is changed : 0.7500

Transformer R/X Ratio $\quad: 0.05000$ 
$\mid * * * * *$ PRESENT WORTH ANALYSIS DATA *****|

Annual Percentage Interest Charges: 15.000

Annual Percent Operation \& Maintenance Charges : 4.000

Life of Equipment (Years) $\quad: 20.000$

Energy Unit Charge (kWh) : $\quad$ : $2.500 \mathrm{Rs}$

Loss Load Factor $\quad: 0.300$

Cost Per MVAr (Lakhs) $\quad$ : $5.000 \mathrm{Rs}$

| SYSTEM DATA |

$\mid * * * * *$ BUS DATA $* * * * * \mid$

BUS NO. AREA ZONE BUS kV VMIN (p.u.) VMAX (p.u.) NAME

\begin{tabular}{|c|}
\hline 00 \\
\hline
\end{tabular}

$\begin{array}{lllllll}2 & 1 & 1 & 11.000 & 0.950 & 1.050 & \text { Bus2 }\end{array}$

$\begin{array}{lllllll}3 & 1 & 1 & 11.000 & 0.950 & 1.050 & \text { Bus3 }\end{array}$

$\begin{array}{lllllll}4 & 1 & 1 & 11.000 & 0.950 & 1.050 & \text { Bus4 }\end{array}$

$\begin{array}{lllllll}5 & 1 & 1 & 11.000 & 0.950 & 1.050 & \text { Bus5 }\end{array}$
* NODE NAME* MW MVAr MIN MAX STEP NO. NO.

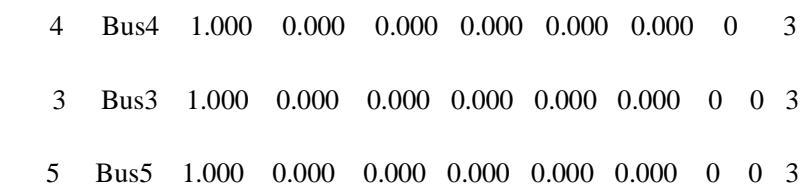

|***** OUTPUT DATA *****|

Total Specified MW Load 3.00000 Changed to 3.00000

Total Specified MVAr Load 0.00000 Changed to 0.00000

Total Specified MVAr Compensation 0.00000 Changed to 0.00000

Total (Including Out of Service Units)

Total Specified MW Generation : 12.00000

Total Minimum MVAr Limit of Generator : 0.00000

Total Maximum MVAr Limit of Generator : 9.00000

Total Specified MW Load $\quad$ : 3.00000 Changed to 3.00000

Total Specified MVAr Load $\quad: \quad 0.00000$ Changed to 0.00000

Total Specified MVAr Compensation : 0.00000 Changed to 0.00000

$\mid * * * * *$ TRANSMISSION LINE DATA $* * * * * \mid$

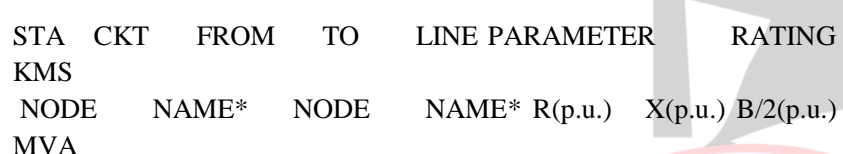

|---- GENERATOR DATA FOR FREQUENCY DEPENDENT LOAD FLOW -----|

SLNO* FROM FROM P-RATE P-MIN P-MAX \%DROOP PARTICI BIAS FACTOR SETTING

$\begin{array}{lrlllllllll}\begin{array}{l}3 \\ 3\end{array} 1 & 1 & \text { Bus1 } & 2 & \text { Bus2 } & 0.21240 & 0.28512 & 0.00000 & 5 \\ \begin{array}{lr}1.00 \\ 3\end{array} & 1 & 2 & \text { Bus2 } & 3 & \text { Bus3 } & 0.21240 & 0.28512 & 0.00000 & 5 \\ 1.00 & & & & & & & & \\ 3 & 1 & 3 & \text { Bus3 } & 4 & \text { Bus4 } & 0.21240 & 0.28512 & 0.00000 & 5 \\ 1.00 & & & & & & & & \\ 3 & 1 & 4 & \text { Bus4 } & 5 & \text { Bus5 } & 0.21240 & 0.28512 & 0.00000 & 5 \\ 1.00 & & & & & & & & \end{array}$

Total Line Charging Susceptance (p.u.) 0.00002

Total Line Charging MVAr at 1 p.u. Voltage Number of Lines Opened on Both the Ends

Total Line Charging susceptance of Existing Lines (p.u.) : 0.00002

Total Line Charging MVAr at 1 p.u. Voltage of Existing Lines: 0.002

Total Capacitive Susceptance $\quad: 0.00000$ p.u. - $0.000 \mathrm{MVAr}$

Total Inductive Susceptance $\quad$ : 0.00000 p.u. - $0.000 \mathrm{MVAr}$

$\mid * * * * *$ GENERATOR DATA $* * * * * \mid$

S1.No* FROM FROM REAL Q-MIN Q-MAX V-SPEC

CAP. MVA STAT

NODE NAME*POWER (MW) MVAr MVAr p.u. CURV RATING

$\begin{array}{llllllllll}1 & 5 & \text { Bus5 } & 6.0000 & 0.0000 & 4.5000 & 1.0000 & 0 & 7.50 & 3\end{array}$

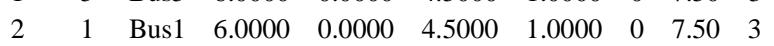

$\begin{array}{lllllllll}1 & 5 & \text { Bus5 } & 6.000 & 0.0000 & 6.0000 & 4.0000 & 0.0000 & 0.0000\end{array}$ $\begin{array}{lll}0.0000 & 0.0000 & 0.0000\end{array}$

$\begin{array}{lllllllll}2 & 1 & \text { Bus1 } & 6.000 & 0.0000 & 6.0000 & 4.0000 & 0.0000 & 0.0000\end{array}$ $\begin{array}{lll}0.0000 & 0.0000 & 0.0000\end{array}$

Slack Bus Angle (degree) : 0.00

$$
\text { OUTPUT RESULTS | }
$$

TOTAL NUMBER OF ISLANDS IN THE GIVEN SYSTEM $\quad: 1$ TOTAL NUMBER OF ISLANDS HAVING ATLEAST ONE GENERATOR: 1

---- SLACK BUSES CONSIDERED FOR THE STUDY -----|

ISLAND NO. SLACK BUS NAME SPECIFIED MW

$$
1 \quad 1 \quad \text { Bus1 } 6.000
$$

|---- CONVERGENCE INDEX -----|

ITERATION BUS MAXP BUS MAX Q

COUNT NUMBER PER UNIT NUMBER PER UNIT

$\begin{array}{lllll}1 & 5 & 0.050 & 4 & 0.005 \\ 2 & 5 & 0.013 & 4 & 0.000 \\ 3 & 5 & 0.004 & 2 & 0.000 \\ 4 & 5 & 0.001 & 2 & 0.000 \\ 5 & 5 & 0.000 & 5 & 0.025 \\ 6 & 5 & 0.023 & 5 & 0.010 \\ 7 & 5 & 0.001 & 5 & 0.000\end{array}$

Number of P Iterations: 5 and Number of Q Iterations: 6

$\mid * * * * *$ BUS VOLTAGES AND POWERS $* * * * * \mid$

SI.No. FROM REAL REACTIVE COMP COMPENSATING

MVAR VALUE CHAR F/V 
NODE FROM V-MAG ANGLE MW MVAr MW MVAr
NO. NAME p.u. DEGREE GEN GEN LOAD LOAD COMP

$\begin{array}{llllllll}1 & \text { Bus1 1.0000 } & 0.00 & -2.858 & 0.174 & 0.000 & 0.000 & 0.000 \\ 2 & \text { Bus2 1.0056 } & 0.49 & 0.000 & 0.000 & 0.000 & 0.000 & 0.000 \\ 3 & \text { Bus3 1.0113 } & 0.96 & 0.000 & 0.000 & 1.000 & 0.000 & 0.000 \\ 4 & \text { Bus4 1.0192 } & 1.59 & 0.000 & 0.000 & 1.000 & 0.000 & 0.000 \\ 5 & \text { Bus5 } 1.0293 & 2.35 & 6.000 & 0.000 & 1.000 & 0.000 & 0.000\end{array}$

NUMBER OF BUSES EXCEEDING MINIMUM VOLTAGE LIMIT (@ mark): 0

NUMBER OF BUSES EXCEEDING MAXIMUM VOLTAGE LIMIT (\# mark): 0

NUMBER OF GENERATORS EXCEEDING MINIMUM Q LIMIT (< mark): 0

NUMBER OF GENERATORS EXCEEDING MAXIMUM Q LIMIT (> mark): 0

|****** LINE FLOWS AND LINE LOSSES $* * * * * \mid$

SLNO CS FROM FROM TO TO FORWARD LOSS \% NODE NAME NODE NAME MW MVAr MW MVAr LOADING

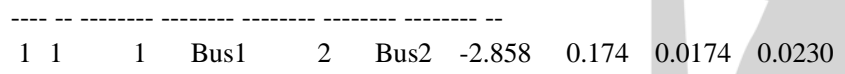

$62.9 \$$

$\begin{array}{llllllllll}2 & 1 & 2 & \text { Bus2 } & 3 & \text { Bus3 } & -2.864 & 0.140 & 0.0173 & 0.0228\end{array}$

$62.6 \$$

31

83.9\#

$\begin{array}{llllllllll}4 & 1 & 4 & \text { Bus4 } & 5 & \text { Bus5 } & -4.855 & 0.038 & 0.0482 & 0.0643\end{array}$ 104.6@

! NUMBER OF LINES LOADED BEYOND 125\%

@ NUMBER OF LINES LOADED BETWEEN 100\% AND 125\%: 1

\# NUMBER OF LINES LOADED BETWEEN 75\% AND 100\%: 1

\$ NUMBER OF LINES LOADED BETWEEN 50\% AND 75\%: 2

$\wedge$ NUMBER OF LINES LOADED BETWEEN 25\% AND 50\%: 0

\& NUMBER OF LINES LOADED BETWEEN 1\% AND 25\%: 0

$*$ NUMBER OF LINES LOADED BETWEEN $0 \%$ AND $1 \%$ : 0

BUSES BETWEEN WHICH ANGLE DIFFERENCE IS > 30 degrees IS: ZERO

ISLAND FREQUENCY SLACK-BUS CONVERGED (1)

$150.00000 \quad 1 \quad 1$

|***** SUMMARY OF RESULTS $* * * * * \mid$

TOTAL REAL POWER GENERATION (CONVENTIONAL)

$6.000 \mathrm{MW}$

TOTAL REAL POWER INJECTION (-ve LOAD)

$0.000 \mathrm{MW}$

TOTAL REACT. POWER GENERATION (CONVENTIONAL) 0.174 MVAr

GENERATION p.f. : 1.000

TOTAL REAL POWER GENERATION (WIND) $0.000 \mathrm{MW}$
TOTAL REACT. POWER GENERATION (WIND) $0.000 \mathrm{MVAr}$

TOTAL REAL POWER GENERATION (SOLAR) $0.000 \mathrm{MW}$

TOTAL REACT. POWER GENERATION (SOLAR) $0.000 \mathrm{MVAr}$

TOTAL SHUNT REACTOR INJECTION

TOTAL SHUNT REACTOR INJECTION

TOTAL SHUNT CAPACIT.INJECTION

TOTAL SHUNT CAPACIT.INJECTION

TOTAL TCSC REACTIVE DRAWL

TOTAL SPS REACTIVE DRAWL

TOTAL UPFC INJECTION

TOTAL SHUNT FACTS INJECTION

TOTAL SHUNT FACTS DRAWAL

TOTAL REAL POWER LOAD

TOTAL REAL POWER DRAWAL (-ve gen.)

TOTAL REACTIVE POWER LOAD

LOAD p.f.

TOTAL COMPENSATION AT LOADS

TOTAL HVDC REACTIVE POWER

TOTAL REAL POWER LOSS (AC+DC) $(0.113834+0.000000)$

PERCENTAGE REAL LOSS (AC+DC)

TOTAL REACTIVE POWER LOSS

$: \quad-0.000 \mathrm{MW}$
$-0.000 \mathrm{MVAr}$
$-0.000 \mathrm{MW}$
$-0.000 \mathrm{MVAr}$
$0.000 \mathrm{MVAr}$
$0.000 \mathrm{MVAr}$
$-0.000 \mathrm{MVAr}$
$0.000 \mathrm{MVAr}$
$0.000 \mathrm{MVAr}$
$3.000 \mathrm{MW}$
$: \quad 2.858 \mathrm{MW}$
$0.000 \mathrm{MVAr}$
1.000
$0.000 \mathrm{MVAr}$
$0.000 \mathrm{MVAr}$
$: \quad 0.113834 \mathrm{MW}$
$: \quad 1.897$
$: \quad 0.151154 \mathrm{MVAr}$

|---- ZONE WISE DISTRIBUTION -----|

Description Zone \# 1

MW generation $\quad 3.1420$

MVAr generation $\quad 0.1742$

MW wind gen. $\quad 0.0000$

MVAr wind gen. $\quad 0.0000$

MW solar gen. $\quad 0.0000$

MVAr solar gen. $=0.0000$

MW load 3.0000

MVAr load 0.0000

MVAr compensation 0.0000

MW loss 0.1138

MVAr loss 0.1512

MVAr - inductive $\quad 0.0000$

MVAr - capacitive $\quad 0.0000$

|-- AREA WISE DISTRIBUTION ----|

Description Area \# 1

$\begin{array}{ll}\text { MW generation } & 3.1420 \\ \text { MVAr generation } & 0.1742 \\ \text { MW wind gen. } & 0.0000 \\ \text { MVAr wind gen. } & 0.0000 \\ \text { MW solar gen. } & 0.0000 \\ \text { MVAr solar gen. } & 0.0000 \\ \text { MW load } & 3.0000 \\ \text { MVAr load } & 0.0000 \\ \text { MVAr compensation } & 0.0000 \\ \text { MW loss } & 0.1138 \\ \text { MVAr loss } & 0.1512 \\ \text { MVAr - inductive } & 0.0000 \\ \text { MVAr - capacitive } & 0.0000\end{array}$




\section{GRAPH OF TOTALHARMONIC DISTOTRION}

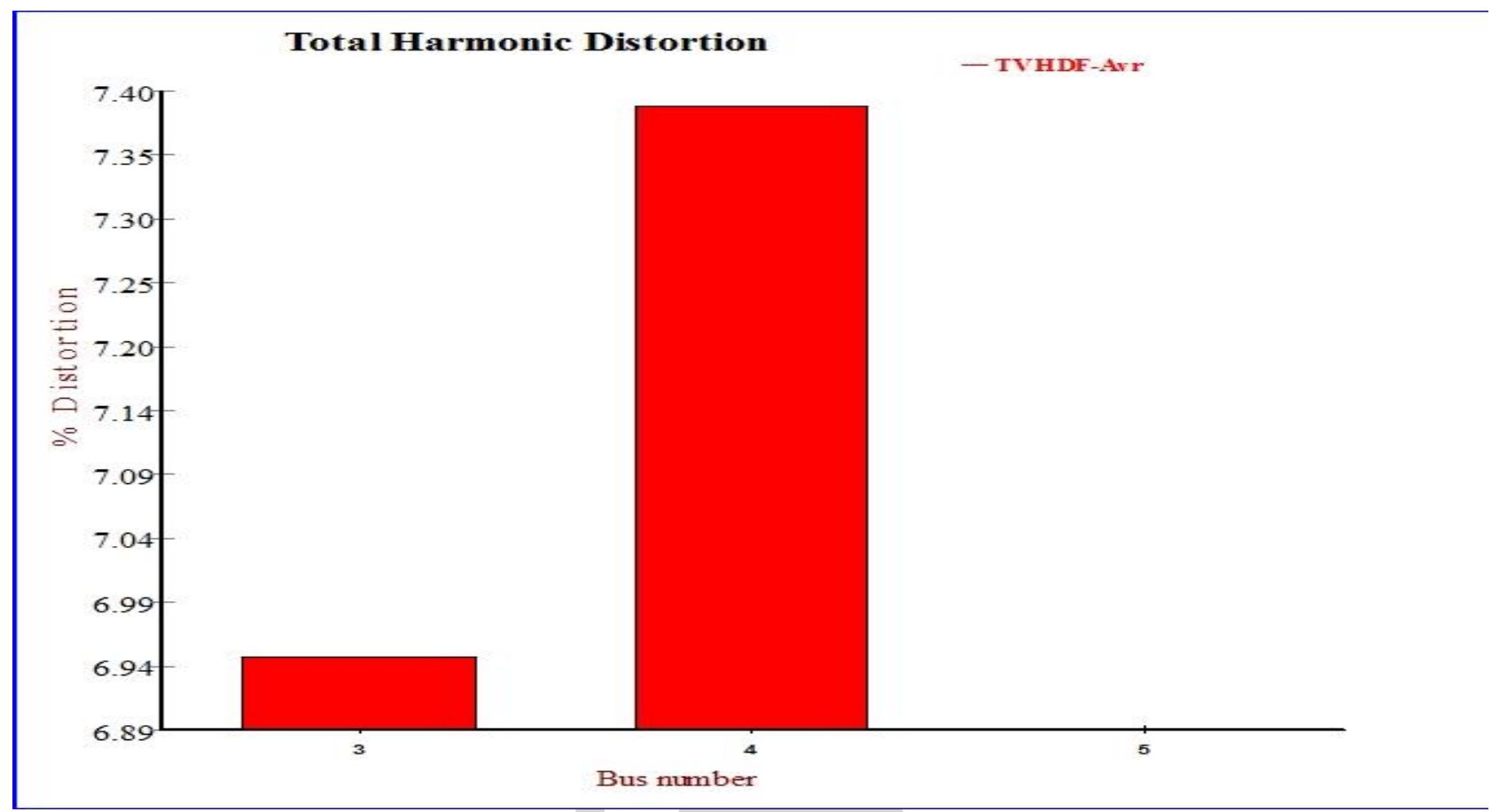

Figure 2 Total Harmonic Distortion

HARMONIC ANALYSIS

CASE NO :

SCHEDULE NO : 0

$\% \%$ First Power System Network

LARGEST BUS NUMBER USED : 5

ACTUAL NUMBER OF BUSES : 5

NUMBER OF ELEMENTS IN RX_B2 FORMAT: 4

NUMBER OF GENERATORS

NUMBER OF LOADS

: 2

BASE MVA 100.000

NOMINAL SYSTEM FREQUENCY : 50.000

INITIAL VOLTAGE OF 1 P.U. IS ASSUMED

NUMBER OF ZONES $\quad: 1$

PRINT OPTION : 3

BOTH DATA AND RESULT APPEAR IN THE REPORT FILE

PLOT OPTION

1

PLOT FILES ARE GENERATED

NUMBER OF HARMONIC ORDERS: 5

HARMONIC ORDERS : $\begin{array}{lllll}2 & 3 & 4 & 5 & 6\end{array}$

NUMBER OF BUSES OF INTEREST: 3 BUS NUMBERS

: 345

TRANSFOMRER R/X RATIO : : 0.05000

CIRCUIT BREAKER R-PU $\quad: 0.00000$

CIRCUIT BREAKER X-PU : 0.00010

NUMBER OF LINE TYPES $3 \times 3 \quad: 0$

NUMBER OF LINE TYPES 6x6 : 0

NUMBER OF LINE TYPES 12x12 : 0
BUSNO STATUS ZONE BUSVOLT KV BUS NAME VOLT-MAG ANG-DEG

$\begin{array}{ccccccc}1 & 1 & 1 & 11.000 & \text { Bus1 } & 1.0000 & 0.000 \\ 2 & 1 & 1 & 11.000 & \text { Bus2 } & 1.0000 & 0.000 \\ 3 & 1 & 1 & 11.000 & \text { Bus3 } & 1.0000 & 0.000 \\ 4 & 1 & 1 & 11.000 & \text { Eus4 } & 1.0000 & 0.000 \\ 5 & 1 & 1 & 11.000 & \text { Bus5 } & 1.0000 & 0.000\end{array}$

$\mathrm{R}, \mathrm{X}, \mathrm{B} / 2$ DATA

STATUS FROM FROM TO TO POSITIVE/ZERO BUS NAME BUS NAME R(P.U) X(P.U.) B/2(P.U) LENGTH

$\begin{array}{lllllll}3 & & & & & & \end{array}$ 0.000001 .00000

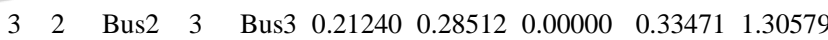
0.000001 .00000

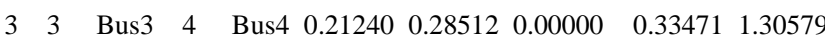
0.000001 .00000

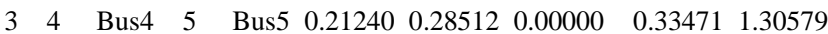
0.000001 .00000

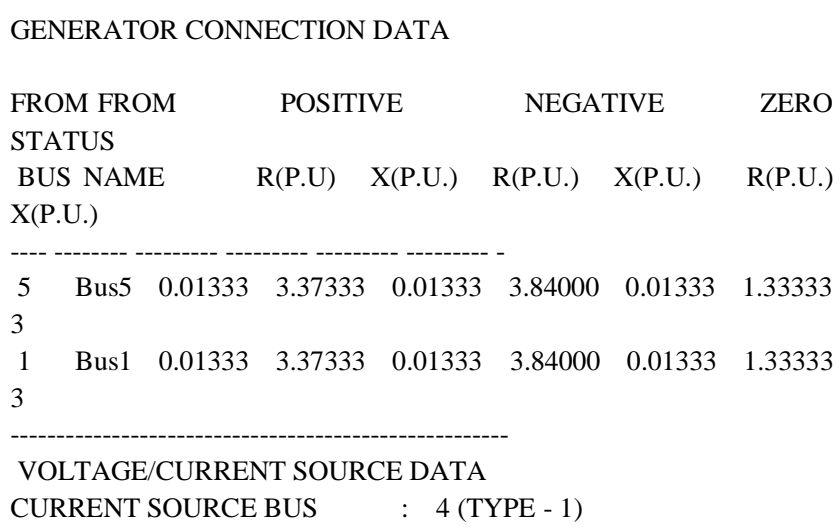


COUNT PHASE A PHASE B PHASE C AMPRMS ANGLE-DEG AMP-RMS ANGLE-DEG AMP-RMS ANGLEDEG

$\begin{array}{ccccccc}2 & 28.169 & 0.000 & 28.169 & 120.000 & 28.169 & 240.000 \\ 3 & 32.193 & 0.000 & 32.193 & 120.000 & 32.193 & 240.000 \\ 4 & 16.096 & 0.000 & 16.096 & 120.000 & 16.096 & 240.000 \\ 5 & 24.144 & 0.000 & 24.144 & 120.000 & 24.144 & 240.000 \\ 6 & 0.000 & 0.000 & 0.000 & 120.000 & 0.000 & 240.000\end{array}$

BUS A-P-MW A-Q-MVAR B-P-MW B-QMVAR C-P-MW CQ-MVAR MD STATUS

$\begin{array}{llllllll}4 & 0.333 & 0.000 & 0.333 & 0.000 & 0.333 & 0.0000 & 3 \\ 3 & 0.333 & 0.000 & 0.333 & 0.000 & 0.333 & 0.0000 & 3 \\ 5 & 0.333 & 0.000 & 0.333 & 0.000 & 0.333 & 0.0000 & 3\end{array}$

INJECTED 2th HARMONIC CURRENT IN AMPs AND DEGREE FOR A, B AND C PHASES

BUS NAME C-A-AMP A-A-DEGR C-B-AMP A-B-DEGR C-CAMP A-C-DEGR

1 Bus1 $0.000-90.000 \quad 0.000 \quad-90.000 \quad 0.000 \quad-90.000$

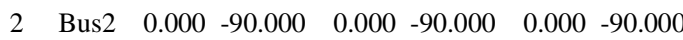

3 Bus3 $0.000 \quad-90.000 \quad 0.000 \quad-90.000 \quad 0.000 \quad-90.000$

$4 \begin{array}{llllllll} & \text { Bus4 } & 28.169 & 0.000 & 28.169 & 120.000 & 28.169 & -120.000\end{array}$

5 Bus5 $\quad 0.000 \quad-90.000 \quad 0.000 \quad-90.000 \quad 0.000 \quad-90.000$

2th HARMONIC BUS VOLTAGES IN VOLTS-DEGREE FOR A, B AND C PHASES

BUS NAME V-A-VOLT V-A-DEGR V-B-VOLT V-B-DEGR V-CVOLT V-C-DEGR

$1 \quad$ Bus1 $122.332 \quad 91.177 \quad 122.340-148.826 \quad 122.341 \quad-28.821$

2 Bus2 $131.464 \quad 89.709 \quad 131.473-150.293 \quad 131.473 \quad-30.289$

$3 \begin{array}{lllllllll} & \text { Bus3 } & 140.671 & 88.433 & 140.680 & -151.569 & 140.679 & -31.565\end{array}$

$4 \begin{array}{llllllll} & \text { Bus4 } & 149.938 & 87.314 & 149.947 & -152.688 & 149.946 & -32.684\end{array}$

$5 \quad$ Bus5 $139.522 \quad 88.781 \quad 139.531-151.221 \quad 139.531 \quad-31.216$

2th HARMONIC LINE FLOWS IN AMPS-DEGREE FOR A, B AND C PHASES

FROM NAME TO NAME I-A-AMP I-A-DEGR I-B-AMP I-BDEGR I-C-AMP I-C-DEGR

1 Bus1 2 Bus2 $13.165-178.724 \quad 13.165 \quad-58.724 \quad 13.165 \quad 61.276$ $\begin{array}{llllll}13.164 & 1.276 & 13.164 & 121.276 & 13.164 & -118.724\end{array}$

2 Bus2 3 Bus3 $13.164-178.724 \quad 13.164 \quad-58.724 \quad 13.164 \quad 61.276$

$\begin{array}{llllll}13.163 & 1.276 & 13.163 & 121.276 & 13.163 & -118.724\end{array}$

$3 \quad$ Bus3 $4 \quad$ Bus4 $13.163-178.724 \quad 13.163 \quad-58.724 \quad 13.163 \quad 61.276$

$\begin{array}{llllll}13.162 & 1.277 & 13.162 & 121.276 & 13.162 & -118.723\end{array}$

4 Bus4 5 Bus5 $15.014 \quad-1.119 \quad 15.014 \quad 118.881 \quad 15.014-121.119$ $15.015178 .88115 .015-61.11915 .014 \quad 58.881$

2th HARMONIC SHUNT INJECTIONS IN AMPS-DEGREE FOR A, B AND C PHASES

BUS NAME I-A-AMP I-A-DEGR I-B-AMP I-B-DEGR I-C-AMP IC-DEGR

$5 \quad$ Bus5 $\quad 15.015 \quad 178.881 \quad 15.015 \quad-61.119 \quad 15.015 \quad 58.881$

1 Bus1 $13.165-178.724 \quad 13.165 \quad-58.724 \quad 13.165 \quad 61.276$

4 Bus4 $0.000 \quad-90.000 \quad 0.000 \quad-90.000 \quad 0.000 \quad-90.000$

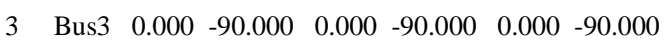

5 Bus5 $0.000 \quad-90.000 \quad 0.000 \quad-90.000 \quad 0.000 \quad-90.000$

INJECTED 3th HARMONIC CURRENT IN AMPs AND DEGREE FOR A, B AND C PHASES

BUS NAME C-A-AMP A-A-DEGR C-B-AMP A-B-DEGR C-CAMP A-C-DEGR

1 Bus1 $0.000 \quad-90.000 \quad 0.000 \quad-90.000 \quad 0.000 \quad-90.000$

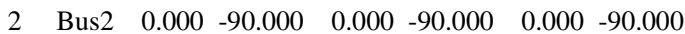

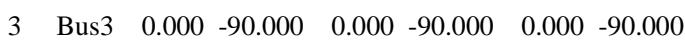

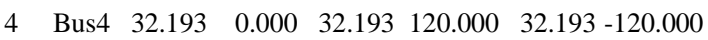

5 Bus5 $\quad 0.000 \quad-90.000 \quad 0.000 \quad-90.000 \quad 0.000 \quad-90.000$

3th HARMONIC BUS VOLTAGES IN VOLTS-DEGREE FOR A, B AND C PHASES

BUS NAME V-A-VOLT V-A-DEGR V-B-VOLT V-B-DEGR V-CVOLT V-C-DEGR

$1 \quad$ Bus1 $209.849 \quad 90.785 \quad 209.864-149.218 \quad 209.864 \quad-29.213$

2 Bus2 $225.468 \quad 89.806 \quad 225.483-150.196 \quad 225.483 \quad-30.192$

3 Bus3 $241.141 \quad 88.955 \quad 241.156-151.047 \quad 241.156-31.043$

4 Bus4 $256.858 \quad 88.207 \quad 256.873-151.794 \quad 256.873-31.790$

5 Bus5 $239.065 \quad 89.186 \quad 239.080-150.816 \quad 239.080 \quad-30.812$

3th HARMONIC LINE FLOWS IN AMPS-DEGREE FOR A, B AND C PHASES

FROM NAME TO NAME I-A-AMP I-A-DEGR I-B-AMP I-BDEGR I-C-AMP I-C-DEGR

$1 \quad$ Bus1 $2 \quad$ Bus2 $15.055-179.149 \quad 15.055 \quad-59.149 \quad 15.055 \quad 60.851$ $\begin{array}{llllll}15.053 & 0.851 & 15.053 & 120.851 & 15.053 & -119.149\end{array}$

2 Bus2 3 Bus3 $15.053-179.149 \quad 15.053 \quad-59.149 \quad 15.053 \quad 60.851$ $\begin{array}{lllllll}15.050 & 0.851 & 15.050 & 120.851 & 15.050 & -119.148\end{array}$

3 Bus3 4 Bus4 $15.050-179.149 \quad 15.050 \quad-59.149 \quad 15.050 \quad 60.852$ $\begin{array}{llllll}15.048 & 0.852 & 15.048 & 120.852 & 15.048 & -119.148\end{array}$

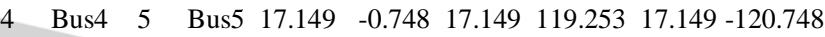

$\begin{array}{llllll}17.151 & 179.252 & 17.151 & -60.747 & 17.151 & 59.252\end{array}$

3th HARMONIC SHUNT INJECTIONS IN AMPS-DEGREE FOR A, B AND C PHASES

BUS NAME I-A-AMP I-A-DEGR I-B-AMP I-B-DEGR I-C-AMP IC-DEGR

\footnotetext{
$5 \quad$ Bus5 $17.151 \quad 179.252 \quad 17.151 \quad-60.747 \quad 17.151 \quad 59.252$

1 Bus1 $15.055-179.149 \quad 15.055 \quad-59.149 \quad 15.055 \quad 60.851$

4 Bus4 $0.000 \quad-90.000 \quad 0.000 \quad-90.000 \quad 0.000 \quad-90.000$

3 Bus3 $0.000 \quad-90.000 \quad 0.000 \quad-90.000 \quad 0.000 \quad-90.000$

5 Bus5 $0.000 \quad-90.000 \quad 0.000 \quad-90.000 \quad 0.000 \quad-90.000$
}

INJECTED 4th HARMONIC CURRENT IN AMPs AND DEGREE FOR A, B AND C PHASES

BUS NAME C-A-AMP A-A-DEGR C-B-AMP A-B-DEGR C-CAMP A-C-DEGR

$\begin{array}{llllllll}1 & \text { Bus1 } & 0.000 & -90.000 & 0.000 & -90.000 & 0.000 & -90.000 \\ 2 & \text { Bus2 } & 0.000 & -90.000 & 0.000 & -90.000 & 0.000 & -90.000 \\ 3 & \text { Bus3 } & 0.000 & -90.000 & 0.000 & -90.000 & 0.000 & -90.000 \\ 4 & \text { Bus4 } & 16.096 & 0.000 & 16.096 & 120.000 & 16.096 & -120.000 \\ 5 & \text { Bus5 } & 0.000 & -90.000 & 0.000 & -90.000 & 0.000 & -90.000\end{array}$

4th HARMONIC BUS VOLTAGES IN VOLTS-DEGREE FOR A, B AND C PHASES

BUS NAME V-A-VOLT V-A-DEGR V-B-VOLT V-B-DEGR V-CVOLT V-C-DEGR

$1 \quad$ Bus1 $139.954 \quad 90.593 \quad 139.964-149.410 \quad 139.964 \quad-29.405$

2 Bus2 $150.359 \quad 89.854 \quad 150.369-150.148 \quad 150.369 \quad-30.144$

$3 \quad$ Bus3 $160.782 \quad 89.211 \quad 160.793-150.791 \quad 160.792 \quad-30.787$

4 Bus4 $171.221 \quad 88.647 \quad 171.231-151.355 \quad 171.231 \quad-31.351$

$5 \quad$ Bus5 $159.372 \quad 89.386 \quad 159.382-150.617 \quad 159.382 \quad-30.612$

4th HARMONIC LINE FLOWS IN AMPS-DEGREE FOR A, B AND C PHASES

FROM NAME TO NAME I-A-AMP I-A-DEGR I-B-AMP I-BDEGR I-C-AMP I-C-DEGR

$1 \quad$ Bus1 2 Bus2 $7.530-179.358 \quad 7.530 \quad-59.358 \quad 7.530 \quad 60.642$ $\begin{array}{llllll}7.529 & 0.642 & 7.529 & 120.642 & 7.529 & -119.357\end{array}$

2 Bus2 $3 \quad$ Bus3 $7.528-179.358 \quad 7.528 \quad-59.358 \quad 7.528 \quad 60.643$

$\begin{array}{llllll}7.526 & 0.643 & 7.526 & 120.643 & 7.526 & -119.357\end{array}$

$\begin{array}{lllllllll}3 & \text { Bus3 } 4 & \text { Bus4 } & 7.526 & -179.357 & 7.526 & -59.357 & 7.526 & 60.643\end{array}$

$\begin{array}{llllll}7.524 & 0.643 & 7.524 & 120.643 & 7.524 & -119.357\end{array}$ 
$\begin{array}{llllllllll}4 & \text { Bus4 } & 5 & \text { Bus5 } & 8.573 & -0.565 & 8.573 & 119.436 & 8.573 & -120.565\end{array}$ $\begin{array}{llllll}8.575 & 179.435 & 8.575 & -60.565 & 8.575 & 59.435\end{array}$

4th HARMONIC SHUNT INJECTIONS IN AMPS-DEGREE FOR A, B AND C PHASES

BUS NAME I-A-AMP I-A-DEGR I-B-AMP I-B-DEGR I-C-AMP IC-DEGR

$\begin{array}{llllllll}5 & \text { Bus5 } & 8.575 & 179.435 & 8.575 & -60.565 & 8.575 & 59.435\end{array}$

$1 \quad$ Bus1 $7.531-179.358 \quad 7.531 \quad-59.358 \quad 7.531 \quad 60.642$

$4 \begin{array}{cccccccc} & \text { Bus4 } & 0.000 & -90.000 & 0.000 & -90.000 & 0.000 & -90.000\end{array}$

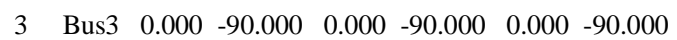

$\begin{array}{llllllll}5 & \text { Bus5 } & 0.000 & -90.000 & 0.000 & -90.000 & 0.000 & -90.000\end{array}$

INJECTED 5th HARMONIC CURRENT IN AMPs AND DEGREE FOR A, B AND C PHASES

BUS NAME C-A-AMP A-A-DEGR C-B-AMP A-B-DEGR C-CAMP A-C-DEGR

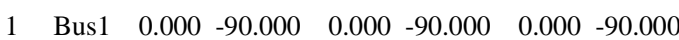

2 Bus2 $0.000 \quad-90.000 \quad 0.000 \quad-90.000 \quad 0.000 \quad-90.000$

$3 \begin{array}{llllllll} & \text { Bus3 } & 0.000 & -90.000 & 0.000 & -90.000 & 0.000 & -90.000\end{array}$

$4 \begin{array}{llllllll} & \text { Bus4 } & 24.144 & 0.000 & 24.144 & 120.000 & 24.144 & -120.000\end{array}$

5 Bus5 $\quad 0.000 \quad-90.000 \quad 0.000 \quad-90.000 \quad 0.000 \quad-90.000$

5th HARMONIC BUS VOLTAGES IN VOLTS-DEGREE FOR A, B AND C PHASES

BUS NAME V-A-VOLT V-A-DEGR V-B-VOLT V-B-DEGR V-CVOLT V-C-DEGR

$1 \quad$ Bus1 $262.518 \quad 90.483 \quad 262.537-149.520 \quad 262.537 \quad-29.515$

2 Bus2 $282.024 \quad 89.882 \quad 282.043-150.121282 .043 \quad-30.116$

$3 \quad$ Bus3 $301.549 \quad 89.359 \quad 301.568-150.643 \quad 301.568$-30.639

4 Bus4 $321.087 \quad 88.900 \quad 321.106-151.102 \quad 321.106-31.098$

$5 \quad$ Bus5 $298.879 \quad 89.501 \quad 298.898-150.502 \quad 298.898$-30.497

5th HARMONIC LINE FLOWS IN AMPS-DEGREE FOR A, B AND C PHASES

FROM NAME TO NAME I-A-AMP I-A-DEGR I-B-AMP I-BDEGR I-C-AMP I-C-DEGR

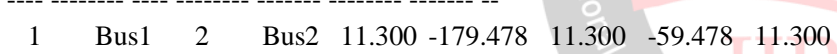
$\begin{array}{lllllll}60.523 & 11.296 & 0.523 & 11.296 & 120.522 & 11.296 & -119.477\end{array}$

$\begin{array}{lllllllll}2 & \text { Bus2 } & 3 & \text { Bus3 } & 11.295 & -179.477 & 11.295 & -59.478 & 11.295\end{array}$ 60.523

$\begin{array}{llllll}11.290 & 0.523 & 11.291 & 120.523 & 11.291 & -119.477\end{array}$

$3 \quad$ Bus3 4 Bus4 $11.290-179.477 \quad 11.290 \quad-59.477 \quad 11.290 \quad 60.523$ $\begin{array}{llllll}11.285 & 0.524 & 11.285 & 120.523 & 11.285 & -119.476\end{array}$

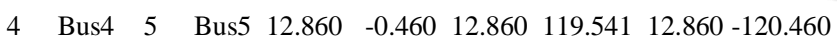
$\begin{array}{llllll}12.865 & 179.540 & 12.865 & -60.460 & 12.865 & 59.540\end{array}$

5th HARMONIC SHUNT INJECTIONS IN AMPS-DEGREE FOR A, B AND C PHASES

BUS NAME I-A-AMP I-A-DEGR I-B-AMP I-B-DEGR I-C-AMP IC-DEGR

$5 \quad$ Bus5 $12.866 \quad 179.540 \quad 12.865 \quad-60.460 \quad 12.865 \quad 59.540$

1 Bus1 $11.300-179.478 \quad 11.300-59.478 \quad 11.300 \quad 60.523$

4 Bus4 $0.000 \quad-90.000 \quad 0.000 \quad-90.000 \quad 0.000 \quad-90.000$

$3 \begin{array}{llllllll} & \text { Bus3 } & 0.000 & -90.000 & 0.000 & -90.000 & 0.000 & -90.000\end{array}$

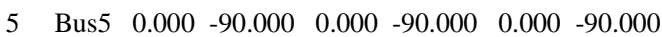

INJECTED 6th HARMONIC CURRENT IN AMPs AND DEGREE FOR A, B AND C PHASES

BUS NAME C-A-AMP A-A-DEGR C-B-AMP A-B-DEGR C-CAMP A-C-DEGR

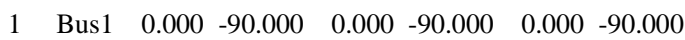

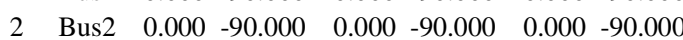

3 Bus3 $\quad 0.000 \quad-90.000 \quad 0.000-90.000 \quad 0.000 \quad-90.000$

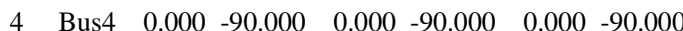

5 Bus5 $\quad 0.000 \quad-90.000 \quad 0.000 \quad-90.000 \quad 0.000 \quad-90.000$ 6th HARMONIC BUS VOLTAGES IN VOLTS-DEGREE FOR A, B AND C PHASES

BUS NAME V-A-VOLT V-A-DEGR V-B-VOLT V-B-DEGR V-CVOLT V-C-DEGR

$\begin{array}{llllllll}1 & \text { Bus1 } & 0.000 & -90.000 & 0.000 & -90.000 & 0.000 & -90.000 \\ 2 & \text { Bus2 } & 0.000 & -90.000 & 0.000 & -90.000 & 0.000 & -90.000 \\ 3 & \text { Bus3 } & 0.000 & -90.000 & 0.000 & -90.000 & 0.000 & -90.000 \\ 4 & \text { Bus4 } & 0.000 & -90.000 & 0.000 & -90.000 & 0.000 & -90.000 \\ 5 & \text { Bus5 } & 0.000 & -90.000 & 0.000 & -90.000 & 0.000 & -90.000\end{array}$

6th HARMONIC LINE FLOWS IN AMPS-DEGREE FOR A, B AND C PHASES

FROM NAME TO NAME I-A-AMP I-A-DEGR I-B-AMP I-BDEGR I-C-AMP I-C-DEGR

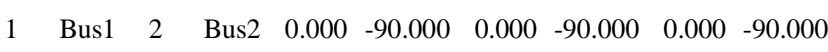
$\begin{array}{llllll}0.000 & -90.000 & 0.000 & -90.000 & 0.000 & -90.000\end{array}$

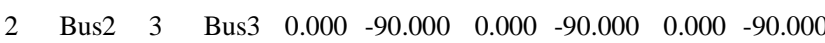
$\begin{array}{llllll}0.000 & -90.000 & 0.000 & -90.000 & 0.000 & -90.000\end{array}$

3 Bus3 4 Bus4 $0.000 \quad-90.000 \quad 0.000 \quad-90.000 \quad 0.000 \quad-90.000$ $\begin{array}{llllll}0.000 & -90.000 & 0.000 & -90.000 & 0.000 & -90.000\end{array}$

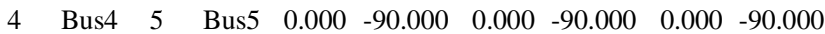
$\begin{array}{llllll}0.000 & -90.000 & 0.000 & -90.000 & 0.000 & -90.000\end{array}$

6th HARMONIC SHUNT INJECTIONS IN AMPS-DEGREE FOR A, B AND C PHASES

BUS NAME I-A-AMP I-A-DEGR I-B-AMP I-B-DEGR I-C-AMP IC-DEGR

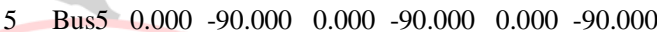

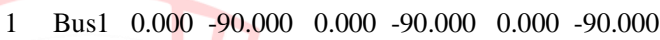

4 Bus4 $0.000 \quad-90.000 \quad 0.000 \quad-90.000 \quad 0.000 \quad-90.000$

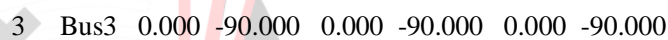

5 Bus5 $0.000 \quad-90.000 \quad 0.000 \quad-90.000 \quad 0.000 \quad-90.000$

VOLTAGE HARMONIC DISTORTION FACTORS

NODE NAME \%HDF-A \%HDF-B \%HDF-C \%HDF-AVR

$1 \quad$ Bus1 $6.0474 \quad 6.0479 \quad 6.0479 \quad 6.0477$

2 Bus2 $6.49726 .4977 \quad 6.4977 \quad 6.4975$

$\begin{array}{llllll}3 & \text { Bus3 } & 6.9482 & 6.9487 & 6.9486 & 6.9485\end{array}$

$4 \begin{array}{llllll}4 & \text { Bus4 } & 7.4001 & 7.4005 & 7.4005 & 7.4004\end{array}$

$\begin{array}{llllll}5 & \text { Bus5 } & 6.8878 & 6.8882 & 6.8882 & 6.8881\end{array}$

INDIVIDUAL VOLTAGE HARMONIC DISTORTION FACTORS NODE NAME $\%$ HDF-T $\%$ HDF- $2 \%$ HDF-3 $\%$ HDF-4 $\%$ HDF- 5 $\%$ HDF-6

$1 \quad$ Bus1 $6.0477 \quad 1.9263 \quad 3.3044 \quad 2.2038 \quad 4.1338 \quad 0.0000$

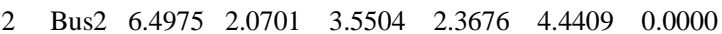

$\begin{array}{lllllllll}3 & \text { Bus3 } & 6.9485 & 2.2151 & 3.7971 & 2.5318 & 4.7484 & 0.0000\end{array}$

$4 \begin{array}{llllllll}4 & \text { Bus4 } & 7.4004 & 2.3610 & 4.0446 & 2.6961 & 5.0560 & 0.0000\end{array}$

$5 \begin{array}{llllllll}5 & \text { Bus5 } & 6.8881 & 2.1970 & 3.7645 & 2.5096 & 4.7063 & 0.0000\end{array}$

TELEPHONE INFLUENCE FACTORS

NODE NAME \%TIF-A \%TIF-B \%TIF-C \%TIF-AVR

$\begin{array}{llllll}1 & \text { Bus1 } & 6215 & 6215 & 6215 & 6215 \\ 2 & \text { Bus2 } & 6677 & 6677 & 6677 & 6677 \\ 3 & \text { Bus3 } & 7139 & 7140 & 7140 & 7140 \\ 4 & \text { Bus4 } & 7603 & 7603 & 7603 & 7603 \\ 5 & \text { Bus5 } & 7077 & 7077 & 7077 & 7077\end{array}$

I-THD and RMS current for series branches FROM NAME TO NAME RMS-HARMONIC(A) FUNDAMENTAL(A) RMS-TOTAL(A) I-THD(\%)

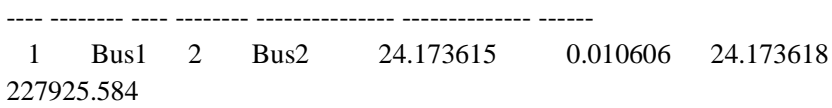




$\begin{array}{ccccccc}2 \quad \text { Bus2 } & 3 & \text { Bus3 } & 24.168743 & 0.010606 & 24.168746 \\ 227879.649 & & & & & \\ 3 \quad \text { Bus3 } & 4 & \text { Bus4 } & 24.163524 & 0.010606 & 24.163526 \\ 227830.438 & & & & & \\ 4 \quad \text { Bus4 } & 5 & \text { Bus5 } & 27.538405 & 0.010606 & 27.538407 \\ 259651.151 & & & & & \end{array}$

\section{CONCLUSION}

This paper carried out the Load flow analysis and Harmonic analysis in a power system. Effect of the harmonics on each bus system is carried out with specified parameters are been studied. This analysis helps us to come to a conclusion on which filter is to be approved to mitigate the harmonics. The results obtained from the software are accurate and precise. This software has given us the results in a short Period.

The total real power generation (conventional) is $6.000 \mathrm{MW}$ of two generators, MW generation is 3.1420 and MW load is 3.0000 and MW loss is 0.1138 . so from above we can conclude that generation is equal to the sum of load and loss. For the Evaluation of Harmonic Analysis five numbers of Harmonic orders are acknowledged, where in harmonic current, harmonic bus voltage, harmonic line flow, harmonic shunt injection are assessed. From the graph, the highest percentage distortion with respect to the bus is ascertained. This is by virtue of RMS current source supplying the harmonics is connected to the Bus system.

\section{REFERENCES}

[1]. Singh, Mandeep, and Sheila Mahapatra. "Implementation of passive filters for harmonics reduction." International Journal of Advanced Science and Technology 78 (2015): 1-12.

[2]. Jairath, A. K. "HARMONIC ANALYSIS OF A POWER DISTRIBUTION SYSTEM" [3]. Patel, Rachana J. "HARMONIC ANALYSIS OF STANDARD IEEE 14 BUS USING PASSIVE FILTER." INTERNATIONAL JOURNAL OF ENGINEERING SCIENCES AND MANAGEMENT: 68.

[4]. Prabhu, Mr A., and Mr A. Subramanian Siva. "Short Circuit and Contingency Analysis Implementation for IEEE-14 Bus System using Mi Power Software." growth 8.5 (2019).

[5]. Ashwini, H. K., Mrutyunjay Mohanty, and CR Sharada Prasad. "Harmonic Analysis in Distribution System Planning."

[6]. Daut, I., et al. "Harmonic Effect on the Three-Phase System Due to Unbalanced Harmonic Current Source Using Load Flow Method." Engineering Conference. 2007.

[7]. Vaikund, Harini, et al. "Power Flow Analysis and Reactive Power Compensation Mi Power-A Case
Study."Analysis and Reactive Power Compensation Mi Power-A Case Study."

[8]. Power Research \& Development Consultants Pvt. Ltd., "Power System Analysis \& Simulation MiPowerTM," Power Research \& Development Consultants Pvt. Ltd., [Online]. 